\title{
Habitat ancien et protection du patrimoine à Hatiheu : une vallée marquisienne
}

\section{Pierre Ottino-Garanger}

\section{(2) OpenEdition \\ 12 Journals}

Édition électronique

URL : http://journals.openedition.org/jso/1616

DOI : 10.4000/jso.1616

ISSN : $1760-7256$

\section{Éditeur}

Société des océanistes

\section{Édition imprimée}

Date de publication : 1 décembre 2001

Pagination : 201-214

ISSN : 0300-953x

\section{Référence électronique}

Pierre Ottino-Garanger, « Habitat ancien et protection du patrimoine à Hatiheu : une vallée

marquisienne », Journal de la Société des Océanistes [En ligne], 113 | Année 2001-2, mis en ligne le 27 mai 2008, consulté le 22 avril 2019. URL : http://journals.openedition.org/jso/1616 ; DOI : 10.4000/ jso. 1616

(c) Tous droits réservés 


\section{Habitat ancien et protection du patrimoine à Hatiheu : une vallée marquisienne}

par

Pierre OTTINO-GARANGER *

\section{RÉSUMÉ}

Associer compréhension de l'histoire d'un peuple et accompagnement de projets locaux en la matière, tel peut être aussi, par moment, la mission et le devoir d'un archéologue. Bien des dérèglements de nos systèmes de vie tiennent à l'aveuglement de préoccupations à sens unique. L'étude des aménagements du territoire de vallées et des clans marquisiens nous a conduit, en particulier, à celle de grands ensembles communautaires $d u$ nord au sud de l'archipel, puis à leur mise en valeur partielle avec des associations locales. Causes et résultats sont ici brièvement exposés.

Mots-clés : archéologie, peuplement, aménagement $\mathrm{du}$ territoire, restauration, patrimoine, tourisme, architecture.

\section{Préambule}

Les Marquises se trouvent dans la partie centrale du triangle de Polynésie orientale, selon la terminologie utilisée par les archéologues, bien qu'elles soient quelque peu décentrées par rapport aux autres archipels « centraux » que sont les îles de la Société, les Tuamotu, les Australes et les Cook. Les Marquises, depuis longtemps, livrent des datations très anciennes, maintes fois

\section{SUMMARY}

To work together upon settlement understanding, for example, with scientific methods and, hand in hand, with local associations whose project lead to the same goal: meaning of the tradition, can be and must be part of archaeologist's preoccupations in certains cicumstances. Many disorders of our present systems of life are the direct consequences of our blindness and stubborn obstinacy for one-way " scientific » goals. Settlement patterns studies of the Marquesan society lead us, through valleys, to pay attention to communities looking forward recognition and economic help; people decided to pull out of vegetation and oblivion remarkable accomplishments of the past for present's sake. Reason and results are briefly exposed in this paper.

Key words : Archaeology, settlement patterns, cultural heritage, restauration.

remises en cause. On passa ainsi de 200 ans avant J.-C., à 400 après J.-C., puis 800 et dernièrement $1200 \ldots$ En caricaturant, les Polynésiens et les Européens seront bientôt arrivés sur ces îles à quelques heures d'intervalle.

Les dates anciennes peuvent et doivent pouvoir être remises en cause mais, à ne prendre en compte que les dernières données d'analyses ponctuelles, on risque de fausser la réalité en confondant une vérité avec l'état actuel des

* Archéologue à l'IRD. 
connaissances. Ces dernières ne sont que partielles et, en refusant d'autres hypothèses, on risque parfois de façonner de fausses certitudes en érigeant en nouvelle vérité des évidences fragmentaires. Par ailleurs, en se focalisant souvent sur ces uniques questions d'ancienneté, on réduit l'archéologie en la vidant d'une bonne part de sa substance. Son intérêt pour l'humain et bien d'autres aspects de l'approche scientifique sont absents. La question paraît bien maigre devant l'ampleur des études à mener pour une meilleure connaissance de ces univers océaniens. C'est, en particulier, le cas face au défi que représente la compréhension des modes de vie, de gestion de l'espace, d'installation et d'aménagement du territoire de populations aussi diverses, parmi elles les anciens Marquisiens. Il faudra encore des années de recherches, d'analyses et d'interrogations pour l'approcher dans toute son ampleur.

Il est certes surprenant que, partis de Polynésie occidentale, les anciens Polynésiens aient touché l'archipel des Marquises en premier, "réussissant » par là même à éviter les nombreuses îles intermédiaires des Cook, de la Société et des Tuamotu. S'il est possible qu'une ou deux pirogues, lors de conditions particulières de navigation, par un trajet au nord de ces archipels, aient réussi à les éviter et à atteindre directement les Marquises, elles ne peuvent suffire à expliquer les voyages d'exploration et l'établissement du peuplement d'un archipel. Il faut envisager un mouvement plus ample, divers allers-retours et ce, sur une assez longue période, sans doute plusieurs siècles. Il est dès lors impossible d'imaginer que les autres archipels aient été ignorés et soient restés inoccupés.

Ces dates anciennes des Marquises ont donc été plus ou moins fortement critiquées dès le début, soit dès 1957. Ces attaques furent plus ou moins vives selon les époques et selon les chercheurs. Si l'on accepte la critique de ces anciennes datations ou que l'on se rende à cette vision d'un peuplement récent de la Polynésie orientale, ce qui est une démarche différente, il faut expliquer bien des points de mise en place et de différenciation culturelle dans un très court laps de temps. Pourquoi les ancêtres des Polynésiens réussirent-ils à reconnaître, en à peine quatre siècles, la plupart des îles s'étendant sur près de $5000 \mathrm{~km}$ d'ouest en est, du nord de la NouvelleGuinée jusqu'à celles des Tonga et des Samoa pour, après une course aussi rapide, se fixer en Polynésie occidentale près d'un millénaire, voire davantage, sans poursuivre au-delà, sur de grands espaces marins certes, mais forts de leurs immenses connaissances des mers et des techniques qui y sont associées. Pourquoi, après cette pause si longue, de nouveaux départs vers l'est et peut-être, plus encore, comment ? En effet, si l'on ne navigue pratiquement plus pendant plusieurs générations, comment repartir en ayant conservé intact l'ensemble des connaissances accumulées sur la construction navale et la navigation. Elles se maintiennent et s'enrichissent par une pratique incessante, comme part intégrante d'une culture, d'une tradition, familière à certains membres au moins des communautés. Toutes ces réflexions, non pas pour maintenir que les Marquises furent seules à être aussi anciennement peuplées, ce qui semble (selon la géographie océanienne et nautique) peu probable, mais pour souligner combien ces modes archéologiques paraissent partiales, insuffisamment raisonnées, lorsqu'elles s'élaborent à partir de convictions personnelles non confrontées aux fruits d'une intensification des recherches et de réflexions croisées.

Enfin, trop souvent aujourd'hui, les recherches en archéologie, malgré une attention plus grande portée aux pays hôtes, s'orientent vers des objectifs étrangers aux préoccupations de ces mêmes pays. Cela s'explique en partie par la façon dont les décisions sont prises. Si les objectifs des uns et des autres ne correspondent pas toujours, il est rare pourtant de ne pas trouver des points de convergence.

\section{Introduction}

Notre mission avait commencé, il y a quelques années, sur une base strictement scientifique, autrement dit avec des objectifs en grande partie extérieurs à la demande locale. Elle s'orienta petit à petit vers une programmation et des enjeux, suscités en partie par des personnalités et responsables locaux aussi simplement que par les sites eux-mêmes et les circonstances. En 1993 avait débuté, à Nuku Hiva, aux îles Marquises, un programme archéologique d'étude des systèmes d'habitat et leurs liens avec le milieu environnant. Il venait compléter un projet de recherche plus vaste, mené depuis un certain temps déjà, sur l'organisation sociale et économique. Hatiheu, importante vallée sur la côte nord de cette île, en est devenue le maillon principal. Elle abrite des vestiges architecturaux et un environnement bien préservé datant, pour l'ensemble le plus tardif, des périodes charnières entre histoire et préhistoire: c'est-à-dire entre l'époque dite classique, pour reprendre la terminologie de Robert Suggs, et celle des débuts de l'implantation européenne : soit entre les $\mathrm{XV}^{\mathrm{e}}$ et $\mathrm{XVIII}{ }^{\mathrm{e}}$ siècles. Ce travail est associé au dépouillement 
de données orales, ethnohistoriques et historiques.

L'origine de la sauvegarde entreprise à Hatiheu est ainsi due à plusieurs facteurs, dont les observations faites par le maire délégué de cette partie de l'île - Yvonne Katupa —, sur la valeur économique et culturelle d'un patrimoine intelligemment mis en valeur, ce qu'elle avait pu réaliser lors de voyages en Nouvelle-Zélande, en France, aux Fidji puis, en 1990-1991, à Hiva Oa. À cela s'ajoutait l'exode des Marquisiens et la prise de conscience qu'au-delà de ces vestiges abandonnés sous la brousse, la vie partait. Cette volonté fut exprimée par les habitants de l'archipel dans des associations, entre artisans, instituteurs ou prêtres réunis en assemblées diverses. Certaines personnalités ressentirent cela plus que d'autres et décidèrent de bouger. Ce fut le cas d'Yvonne Katupa, déjà nommée, ou de Lilianne Teikipupuni, de Tahuata, dont le long séjour à Tahiti au contact des populations des « archipels lointains » mal intégrées, donnait à ses paroles et ses actes une valeur supplémentaire.

\section{La compréhension entre le chercheur et la commu- nauté}

Puamau, sur l'île de Hiva Oa, Hapatoni, à Tahuata, et Hatiheu, à Nuku Hiva, sont autant de vallées (en tant qu'entité morale) qui furent à l'initiative des travaux de recherches et de restaurations qui y furent menés. Chacun aborde, par contre, cette aventure avec une optique légèrement différente, bien que complémentaire. Il est très important de bien s'entendre et se comprendre pour que, de part et d'autre, l'issue et le débat qui s'instaure au fil de l'avancement des recherches et des décisions à prendre, soient satisfaisants pour le patrimoine, la communauté et la recherche!

Dans ces îles, l'archéologue ne peut et ne doit pas travailler isolément. Plus encore lorsque le projet concerne de grands ensembles. Cette question de dimension des sites va en effet de pair avec un gros investissement de temps, ce qui, inévitablement, signifie du temps partagé avec la communauté où l'on se trouve. Ceci est encore plus vrai lorsque la vallée se sent concernée et s'intéresse au projet, ce qui est sympathique et fort stimulant! La difficulté et l'intérêt de ce travail est en effet de répondre à une attente, tout en poursuivant un objectif de recherche... Or, l'archéologie est souvent ressentie, malheureuse- ment à présent, plus ou moins consciemment ou de façon plus ou moins formulée, comme prédatrice. Elle est le fait de gens venus d'ailleurs qui repartent en laissant un vide. Un vide moral dû à l'incompréhension ou à la méconnaissance de ce que l'autre est venu faire; un vide réel, sur le terrain, par la disparition, effective ou supposée, de choses mal cernées, parfois, et que certains avaient pu croire oubliées alors que le simple fait de s'y intéresser les avaient fait renaître, avec plus ou moins d'exactitude d'ailleurs, dans " le dit» de la communauté...

Travaillant depuis longtemps dans ces îles, les Marquisiens attendaient de nous que nous transmettions ou que, tout simplement, nous soyons utiles tels les premiers "beachcombers" échoués sur les rivages marquisiens qui s'intégraient, d'une façon ou d'une autre, à la communauté où ils vivaient en apportant quelque chose. L'hospitalité polynésienne ne dure que quelques jours, après l'hôte doit partir, se rendre utile ou à défaut, finir au moins au four. Ainsi, autrefois, celui qui avait un savoir, une technique, un don... le transmettait. Comme ce que nous savons nous le devons, certes, à notre travail mais aussi beaucoup au pays lui-même, et plus encore à ses habitants, il s'agit d'un échange naturel, d'une sorte de retour, d'un partage! Pour cela il fallut, de part et d'autre, avancer en s'adaptant à l'autre et aux nécessités du terrain. Ceci veut dire pouvoir ou devoir modifier, moduler, accepter, ne pas faire certaines choses et en faire d'autres, pas toujours prévues dans le projet scientifique initial.

\section{Le soutien moral, matériel et financier}

Pour que cette volonté et ces efforts aboutissent, encore fallait-il que le projet soit soutenu, moralement, financièrement ou matériellement, car même s'il est toujours possible de travailler très modestement, au-début, si l'on veut que les choses avancent et qu'effectivement le projet passe d'une intuition valable à une entreprise culturelle, sociale et économique, il faut des relais tenaces. La perspective du festival des arts, associé par la suite à la célébration du passage à l'an 2000, fut la clef qui devait véritablement motiver l'intérêt des grands relais : c'est-à-dire l'État et le Territoire qui renforcèrent sérieusement la participation de la commune de Nuku Hiva et de quelques associations apportant de petites aides et beaucoup d'énergie ${ }^{1}$. Ces travaux n'auraient bien sûr même pas pu être envi-

1. Les personnages clefs de cette merveilleuse combinaison furent quelques personnalités conscientes de l'intérêt de l'enjeu et informées des possibilités que pouvaient apporter des ministères d'État : ceux de la Culture et de l'Outre-Mer et le Territoire. Parmi eux, il faut nommer un journaliste, Jean-Luc Blain, lancé avec passion dans l'aventure d'une radio marquisienne auprès 

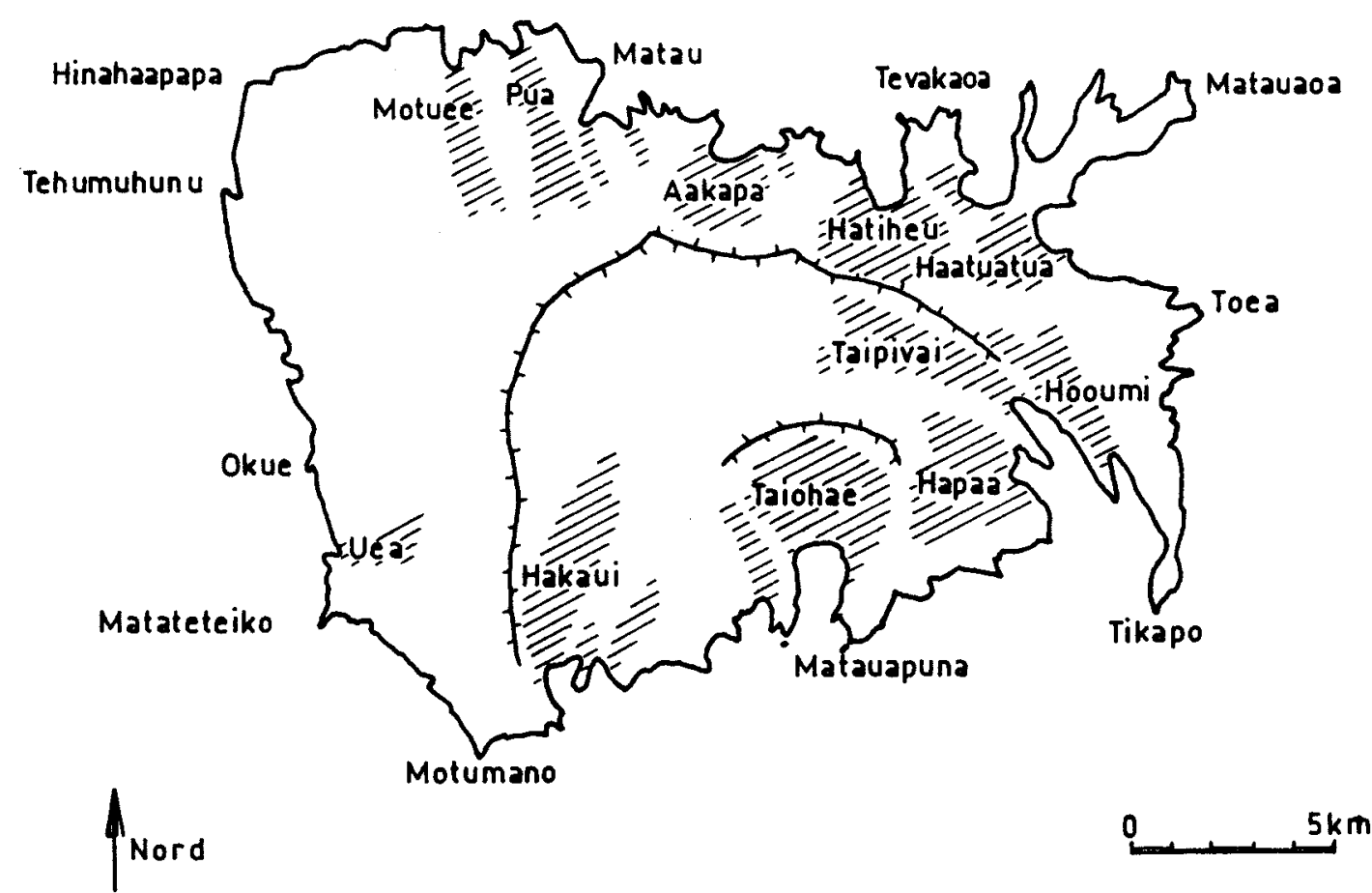

Fig. 1. - Carte de Nuku Hiva.

L'île de Nuku Hiva, de $340 \mathrm{~km}^{2}$, est l'île principale du groupe nord des îles Marquises. Cet archipel polynésien de $1300 \mathrm{~km}^{2}$, à $1500 \mathrm{~km}$ de Tahiti, est composé de treize îles dont huit principales; situé entre $7^{\circ} 50$ et $10^{\circ} 35$ de latitude sud et $138^{\circ} 25$ et $140^{\circ} 50$ de longitude ouest, il s'étire sur $350 \mathrm{~km}$ selon un axe nord-nord-ouest/ sud-sud-est. Six îles sont actuellement habitées et Taiohae, chef-lieu de Nuku Hiva, est le centre administratif de l'archipel..

sagés si l'Orstom, aujourd'hui IRD, n'avait soutenu ces recherches.

Comment remercier ici tous ceux et celles qui apportèrent leur contribution: les ouvriers municipaux qui construisirent nos engins, les travaux publics et les services de l'Agriculture ou de la Santé, la Mission, les jeunes de Hatiheu et 'A'akapa, ces gens de tous âges de Nuku Hiva et des autres îles, ceux de Polynésie et d'ailleurs, les enfants du Meij, les élèves de Moorea, de Tipaerui et de la Fleur de Lampaul qui apportèrent travail, sourires et bonne humeur. On ne peut pas ne pas avoir une pensée toute particulière pour les militaires du Rimapp (régiments d'infanterie de Marine de Tahiti et de Verdun !) et du SMa de Hiva Oa (à travers ce Service militaire adapté, des hommes de tous les archipels de Polynésie, et leurs adjudants, furent extraordinaires), sans qui ces travaux n'auraient jamais atteint une telle ampleur, dans un tel délai, et qui permirent, avec la Marine, la réalisation de ce festival dans un archipel si éloigné des facilités et des infrastruc- tures habituelles, disponibles ailleurs. À Hatiheu, nous n'avons pas travaillé avec de gros engins ni du reste beaucoup d'archéologues ; les faire venir est coûteux et la Polynésie en manque cruellement de disponibles. Nous avons donc travaillé avec des enfants, des jeunes, quelques adultes, des pêcheurs, des casseurs de coprah, des jardiniers, de jeunes tatoueurs, de jeunes sculpteurs, des buveurs de bière et des fumeurs (tabac bison et paka cannabis) qui tous et toutes ont partagé cette aventure en la menant à bien, du début à la fin.

$\mathrm{Au}$ préalable, il fallut de l'acharnement et déterminer si les structures convenaient à la restauration, par leur état et leur nature, mais aussi leur localisation afin d'éviter des démêlés locaux, par la suite, et que l'accès soit simple à la fois pour la mise en œuvre des travaux et pour l'accès des visiteurs. Ce fut un premier investissement demandant beaucoup d'efforts mais sans comparaison avec ce qui suivit. Deux ensembles importants furent retenus : l'un dans la vallée

d'un maire ambitieux, Lucien Kimitete, et tout un personnel d'État : deux administrateurs, Dominique Cadilhac et Bernard Lesterlin, un Haut-Commissaire, Jean-Michel Jau, et un Secrétaire Général, Michel Jeanjean, un vice-amiral d'escadre, des capitaines et un ministre de la Culture du Territoire, Louise Pelzer; enfin, beaucoup d'hommes et de femmes énergiques et dévoués. Comment ne pas citer le minutieux travail, enthousiaste, d'une vingtaine d'enfants de Nuku Hiva venus à deux reprises, lors de leurs vacances, avec un encadrement de jeunes en formation. 


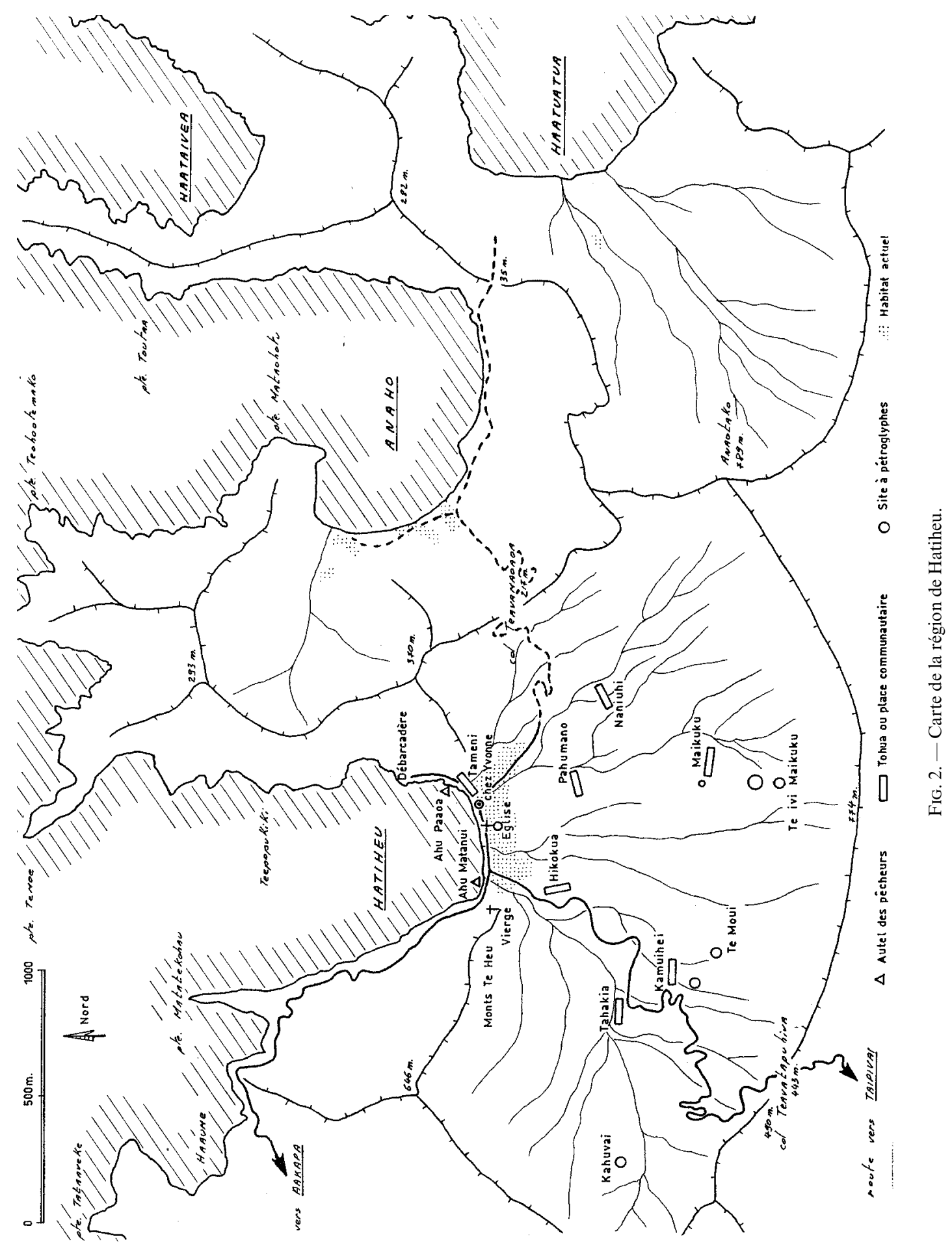


principale de Nuku Hiva, à Taiohae, et l'autre à Hatiheu : celui dont nous parlerons surtout.

\section{La vallée de Hatiheu et les travaux entrepris}

La vallée de Hatiheu, auxquelles étaient associées les vallées de Anaho, Haataivea et Haatuatua, est la plus grande et la mieux arrosée de l'ensemble. L'habitat ancien occupait la presque totalité du territoire de la vallée, en dehors des parties trop en déclivité juste en contrebas des crêtes. Ces dernières étaient par contre utilisées pour des sites mortuaires, de surveillance ou comme lieux de passage pour entrer et sortir de la vallée. La répartition des tohua ou centres communautaires, par leur distribution, donnent idée de l'ancienne extension de l'habitat, d'une étendue et densité sans commune mesure avec l'habitat d'aujourd'hui. Les pétroglyphes, très nombreux, se rencontrent dans toute la vallée et donc bien au-delà de ces principaux sites.

Cette vallée spacieuse de un peu moins de $10 \mathrm{~km}^{2}$ comptait aux temps historiques sept grands tohua (site communautaire ou place des fêtes) : Kamuihei et Tahakia - qui nous intéressent ici - Hikokua et Tameni - ce dernier, sur le littoral, fut détruit par le raz-de-marée de 1946 - , puis Pahumano (ou Pa'aha'ua), Naniuhi et Maikuku. Tous ces tohua, jusque dans la seconde moitié du XIX ${ }^{\mathrm{e}}$ siècle, avaient été les théâtres actifs de la vie des cinq clans Taipi ${ }^{2}$ que comptait encore la vallée. Kamuihei et Tahakia, en amont du village actuel, de part et d'autre de la piste venant de la côte sud - ou vallée principale de Taiohae - , furent choisis comme cadre d'une part des festivités.

La diversité et l'importance des structures environnant ces tohua caractérisent un " centre » assez lâche qui s'étend sur plus de dix hectares. Il fut soigneusement relevé, mais seulement une partie fut retenue pour la mise en valeur ${ }^{3}$. Cet espace concerne majoritairement des terres Puhioho: un clan réputé par son importance démographique et la valeur de ses guerriers, dans la tradition orale. Leur territoire s'étendait de la mer à la montagne et comptait, à l'ouest, une zone de culture, riche et humide, tournée vers l'horticulture en terrasses et l'arboriculture. Elle constituait une sorte de grenier pour la commu- nauté alors que sa puissante cascade, au fond, lui garantissait de l'eau pratiquement sans discontinuer : un atout rare dans l'archipel, par temps de sécheresse ou pire, de disette !

Ce territoire, situé sur les premières pentes au sud-ouest de la vallée, jouait un rôle stratégique et défensif dans le voisinage de clans parfois hostiles. Il était le premier touché lors d'incursions, venant de l'ouest surtout, avec la vallée de 'A'akapa, en partie occupée par un groupe traditionnellement ennemi. Au sud, le risque existait certes, mais à un moindre degré, puisque la vallée de Taipivai était, comme son nom l'indique, habitée par d'autres Taipi, traditionnellement alliés. À l'est, Anaho et Ha'atuatua étaient apparentées à Hatiheu, et en principe alliées. Elles honoraient du reste la même divinité majeure : Tevanauaua qui se détache d'une des dalles rectangulaires (ke'etu), délimitant l'espace carré où étaient présentées les offrandes à Hikoku'a : le premier tohua restauré par la population, à quelques centaines de mètres en aval ${ }^{4}$.

Si l'ensemble mis en valeur se visite agréablement aujourd'hui, il n'en allait pas de même en 1998. Les premiers travaux consistèrent à sortir les structures de leur gangue végétale. La nature, en particulier l'Hibiscus tiliaceus (hau), étouffait tout et en avait éboulé beaucoup. La plupart des essences correspondaient aux besoins de l'ancien centre communautaire et certaines étaient tapu ou sacrées. Les arbres à pain avaient souffert de l'abandon et du remplacement, à certains endroits, par les cocotiers. Il restait en particulier des banians, châtaigniers de Polynésie et bancouliers (Aleurites moluccana) aux côtés des semis des oiseaux : ilang-ilangs et caféiers. Il fallait donc dégager, mais aussi sauvegarder, les témoins du passé et un ombrage aussi nécessaire aux visiteurs et structures qu'à l'avifaune, plus riche ici que sur la côte sud. Elle compte plusieurs endémiques, dont les fauvettes des Marquises et le carpophage de Nuku Hiva, en voie de disparition, et des oiseaux de mer: sternes et pailles-en-queue surtout. Cette tâche fut assurée par des employés municipaux assistés d'emplois jeunes, lorsque c'était possible, leur petit nombre allant en augmentant au fil des mois.

Il fallut parallèlement, et ensuite, procéder aux relevés, puis aux premiers décapages, et enfin aux restaurations avant d'entreprendre la restitution,

2. Branche cadette qui, avec son aînée Tei'i, se partageaient l'île par moitié. Les cinq clans Taipi de Hatiheu étaient les Atikea, Atipuku, Puhioho, Tapatea et Tuuoho.

3. Un travail sur les pétroglyphes y avait été également mené par une doctorante de l'université de Berkeley, S. Millerström, en partenariat avec le Département d'archéologie du Territoire ; nous y avons apporté, hors de ce cadre, un certain nombre d'éléments lors de nos travaux d'étude et de restauration.

4. La divinité principale de Hatiheu était Tauamanaoa mais le site de Te I'ipoka, mis en valeur pour le festival en amont de Kamuihei, était consacré à Tevanauaua. 
d'après documents anciens, de quelques $h a^{\prime} e$ : constructions en matériaux ligneux, recouverts de feuilles ou de palmes, dressées sur les platesformes de pierres. Cela se fit sur un espace de 350 $\mathrm{m}$ sur $350 \mathrm{~m}$, environ, en procédant des structures majeures vers celles qui leurs étaient associées, les restaurations ne s'effectuant donc que sur une partie, soit essentiellement Kamuihei et Tei'ipoka.

Cette étape, du fait de sa richesse, de son étendue et de la végétation, fut longue. La restauration le fut aussi car elle nécessita, pour un petit nombre de personnes, un effort soutenu sur une très longue période, ce qui fut éprouvant ! Ce fut là, probablement, la tâche la plus difficile quoique passionnante pour tous, notamment les responsables, du fait de la fatigue ou du doute lorsque le temps, dans tous les sens du terme, nous jouait des tours (les conséquences d'El Niño se traduisant aux Marquises par des pluies très abondantes en 1998-1999). La présence quotidienne du maire-délégué, qui connaissait parfaitement l'endroit, fut indispensable à la fois pour partager et aiguillonner les efforts, mais aussi en débattre avec l'archéologue qui, de son côté, se devait de partager les tâches les plus variées afin que tous se sentent concernés, et considérés, dans ce travail d'équipe.

\section{Le contexte archéologique}

Aux îles Marquises, les structures élevées sur des paepae (plates-formes lithiques) s'éparpillent du rivage au fond des vallées habitables et jusqu'au sommet des crêtes. La plupart, cependant, se trouvent au centre du territoire, de part et d'autre du cours d'eau principal, à l'abri des incursions ennemies venant de la mer ou des crêtes, mais aussi à l'abri des tsunamis qui ravagent la zone littorale et remontent parfois assez haut. Les Marquisiens s'y regroupaient en hameaux constitués d'unités familiales, dans un espace dégagé, ensoleillé et bien ventilé où se développait favorablement la couverture végétale qui les mettait à l'abri des ardeurs du soleil aussi bien que de la faim.

Les espèces plantées se mêlaient à celles dont on favorisait la croissance en cas de besoin. Les terrasses et jardins de taro, bananiers, patates douces, etc. se succédaient en s'étageant partout où la terre et l'eau pouvaient être associées, même loin des habitations. Les châtaigniers (Inocarpus edulis) de Polynésie, ihi aux Marquises, mape à Tahiti) poussaient dans les zones les plus humides en compagnie souvent d'aracées et les arbres à pain (Artocarpus altilis) : mei ; à Tahiti : uru, maiore), qui fournissaient la base de la nour- riture, couvraient la bonne partie du territoire restant.

Chaque groupe familial ou centre d'activité avait son espace religieux, mais les plus sacrés de la communauté, les me'ae, étaient situés en des lieux soigneusement évités, à l'écart donc des zones de circulation et en général en marge du territoire, tout comme les lieux défensifs ou les sites refuges.

Quant aux espaces communautaires, les places des fêtes (taha koika ou tohua), ils étaient aménagés aux abords des habitations de chefs (ces derniers étant choisis, et éventuellement révoqués, par la communauté). L'espace sacré de cette place était souvent un peu à l'écart, mais « présent » malgré tout sur le petit côté consacré aux prêtres qui faisait face à celui occupé par ceux de « la classe des chefs » ou papa haka'iki. Sacré et profane étaient toujours intimement liés, en effet, dans le quotidien comme lors de festivités. Au cours des siècles, ces espaces dégagés, environnés de bâtiments d'accueil, s'étaient structurés en cours rectangulaires entourées de paepae (plate-forme lithique) et de gradins de mieux en mieux aménagés et de plus en plus vastes.

\section{Le cadre géographique}

Hatiheu est une vallée ouverte sur l'océan par une ample baie frangée de sable noir et de gros galets de basalte. La zone littorale est assez plane et la pente des versants est raisonnable pour cet archipel où les terrains plats sont l'exception; raison du reste de la succession d'aménagements dont ils firent l'objet. À l'arrière, ceux-ci se relèvent progressivement, et plus fortement, pour atteindre une ligne de crête qui culmine entre 500 et $800 \mathrm{~m}$ d'altitude. Ces crêtes délimitent parfaitement la vallée, sans pour autant l'enfermer. Ainsi, à l'est, le col de Anaho, Teavaimaoaoa, ne se situe qu'à $217 \mathrm{~m}$ d'altitude et celui allant vers Taipivai, Teavaitapuhiva, est à moins de $500 \mathrm{~m}$ de hauteur.

De cette crête principale, descendent des arêtes secondaires, que le regard suit jusqu'à la limite des premières pentes, bien en arrière $d u$ littoral où elles se fondent dans un paysage plus homogène, constitué surtout aujourd'hui de cocotiers : ehi et autrefois d'arbres à pain : mei. Ces arêtes délimitent naturellement des territoires arrosés de torrents, intermittents souvent. Elles constituent également des voies d'accès commodes et rapides pour joindre le plateau qui domine le nord-est de l'île, en territoire Taipi, à $700 \mathrm{~m}$ d'altitude environ. Ce plateau, inhabité, était à la fois un lieu de passage, de circulation 
entre vallées et aussi de collecte de plantes rares, ou poussant en altitude.

Les arêtes secondaires sont régulières et leur montée, quoique raide par endroit, est assez progressive sauf au-dessus de la zone qui nous intéresse : Kamuihei. À cet endroit, la roche émerge du paysage en formant un éperon, Te Moui, qui domine la vallée ainsi que la baie. Son sommet est presque horizontal. Ce replat remarquable, le seul de la vallée, fut aménagé pour un guetteur ; une femme s'y illustra, selon la tradition. Elle surveillait le territoire et donnait l'alerte par un cri retentissant; non loin de là, en contrebas, à l'orée du site récemment restauré, se trouve un bâtiment de grande taille où pouvaient se tenir les guerriers prêts à répondre à l'appel, comme en d'autres vallées.

L'ensemble restauré de Kamuihei et Tei'ipoka est installé au pied de ce promontoire. Les rochers, détachés de la paroi, n’y manquent pas et furent mis à profit pour les constructions mais le choix de cet emplacement ne s'explique pas uniquement par cette simple raison. Il faut souligner la valeur symbolique attachée à l'avancée rocheuse qui, comme nombre d'autres, rapprochait les anciens de leurs ancêtres divinisés. Les 'enana ou 'enata, êtres humains originaires de l'archipel, se considéraient issus de Papa, la roche stratifiée. Nombre de vallées avaient leur rocher fondateur. Les Marquisiens répugnaient à enterrer les corps, préférant les conserver près d'eux, puis en confier les os aux racines des banians, aux pierres sèches des paepae ou aux niches funéraires : abrités sous de gros rochers et au creux des falaises.

Ainsi, les parties les plus sacrées de l'espace concerné, les structures funéraires et une très grande part des pétroglyphes se trouvent aux alentours de ce contrefort et, pour une part d'entre elles, se hissent sur ses flancs. Elles assurent ainsi la liaison entre les aménagements plus profanes, en contrebas, et les zones plus hautes, et sans doute plus sacrées, du territoire. Ainsi, une hiérarchie spatiale épouse la topographie de l'endroit.

\section{Présentation du site}

Cet ensemble architectural de TahakiaKamuihei-Tei'ipoka, qui couvre un terrain dont la pente passe de plus de quarante mètres, au nord, à plus de cent-vingt mètres en haut du site, se compose de plusieurs éléments remarquables aux alentours desquels se trouvent d'autres structures liées au quotidien. La répartition et la densité des structures révèlent l'intense occupa- tion de l'espace de cette partie de la vallée. Ici, en partant du bas vers le haut on trouve deux grandes zones mises à profit pour des terrasses irriguées naturellement de part et d'autre du grand tohua de Tahakia (155 m de long sur $45 \mathrm{~m}$ de large). Entre ce dernier et le tohua de Kamuihei (142 $\mathrm{m}$ de long sur $38 \mathrm{~m}$ de large), les jardins, attenants aux paepae ou plates-formes d'habitation, étaient destinés à des cultures plus sèches et qui pouvaient être arrosées grâce à un lit de ruissellement canalisé par des murets de retenue. Juste en face et à l'ouest de Kamuihei, un paepae particulièrement grand ( $20 \mathrm{~m}$ de long sur $12 \mathrm{~m}$ de large) devait servir de lieu de réunion aux chefs et aux guerriers. Il est orienté vers le haut de la pente en direction du me'ae (site religieux) de Te I'ipoka qui le domine et qui est abrité par le plus grand banian de la vallée. Encore plus en hauteur et à l'est du torrent, l'aménagement rectangulaire, qui se présente comme un petit tohua, devait être réservé aux activités religieuses sous la direction des prêtres. Nous sommes ici dans la partie la plus élevée de l'ensemble, juste au pied et sur les contreforts de l'avancée rocheuse de Te Moui qui domine la vallée. En arrière encore et sur la pente même de Te Moui, les structures funéraires les plus tapu (sacrées) ont été établies dans la partie la plus haute et la plus protégée de ce vaste ensemble. De nombreux pétroglyphes ont été piquetés sur de gros rochers en place, surtout dans les zones réservées aux chefs et aux prêtres. De très grandes fosses étaient destinées à ensiler le $m a$ (pâte du fruit de l'arbre à pain) qui était conservé pour la collectivité afin d'être consommé lors des périodes de festivités et de disettes.

Par ailleurs, toute la vallée, et cet endroit en particulier, est constellée de pétroglyphes. Si certains ont été piquetés sur des pierres utilisées en parement ou en pavage des structures lithiques, ils sont habituellement tracés sur des blocs profondément ancrés dans le sol qui n'ont donc pas été déplacés. Certains de ces rochers ornés ont été intégrés aux constructions (comme «le rocher au chien » du long gradin amont de Kamuihei), mais la grande majorité se situe à côté des structures construites. Ils font cependant partie intégrante des aménagements anthropiques, au même titre d'ailleurs que certains végétaux et arbres majestueux.

\section{Les zones basses}

Plus on s'éloigne de l'éperon rocheux de Te Moui, en allant vers le nord, plus on se rapproche de la mer. Cette partie de la vallée est humide car les résurgences sont nombreuses (au nord 


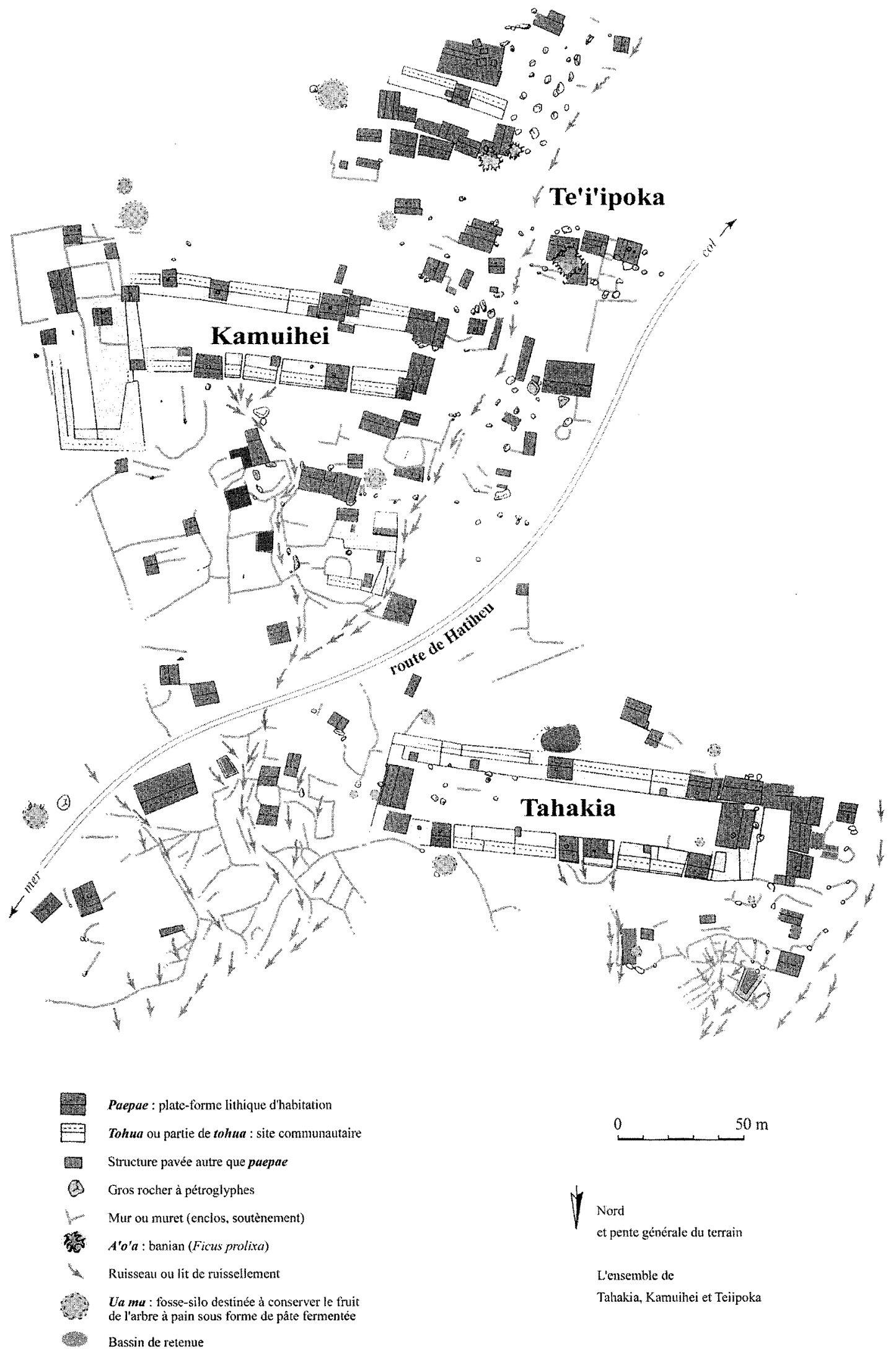

FIG. 3. - L'ensemble de Kamuihei, Tahakia et Te'i'ipoka. 
surtout qui n'a pas été cartographié) ; elles apparaissent déjà à l'est et à l'ouest du tohua Tahakia. Elles transforment en immense jardin inondé, ou facilement irrigable, une vaste dépression couverte d'un réseau serré de murs et murets de retenues. Les paepae d'habitation y sont rares, localisés sur les éminences les plus rocheuses. Cette zone est consacrée, dans son ensemble, à la culture, et fut probablement exploitée de façon communautaire ou destinée à la communauté. Les plantations devaient essentiellement correspondre à des aracées associées aux châtaigniers de Polynésie ; les anguilles pouvaient y trouver un milieu favorable.

Plus en hauteur, les terrains en pente sont moins humides. Ils firent l'objet de nombreux aménagements destinés à des habitations. L'eau ne stagne plus mais s'écoule en petits ruisseaux torrentiels appréciés des crevettes d'eau douce. Elle y est plus canalisée par des murs que freinée ou barrée par des murets destinés à la répartir à travers des terrasses plates, sur un maximum de surface, comme en contrebas. Ici l'eau devait être captée simplement en fonction des besoins des plantes, et les jardins abritaient des types de cultures plus sèches tels bananiers et patates douces, associés, là encore, à une arboriculture sans doute constituée essentiellement d'arbres à pain.

Le terrain est ici plus sec, il se tient donc mieux et n'a pas nécessité d'être façonné comme dans la zone détrempée en contrebas. Les parcelles encloses de murets, non aménagées en terrasses, y sont de plus grandes dimensions que plus bas. Les murets s'appuient souvent sur des paepae en place et les parcelles semblent attenantes aux habitations. Mais cette parcellisation du paysage horticole ne semble pas obéir à des contraintes de "propriété », mais plutôt, et tout simplement, à celles du relief et de la nature du sol.

L'ensemble de cet environnement était probablement largement destiné à la collectivité, comme le laisse également supposer la présence de treize fosses-silos (' $\mathrm{ua} \mathrm{ma}$ ), toutes de grandes dimensions, dont huit particulièrement imposantes. Peu de petits silos de type familial ont été répertoriés ${ }^{5}$.

\section{Les zones hautes}

En se rapprochant du pied de l'avancée rocheuse, la pente devient plus forte. La présence du piton Te Moui est sensible, en particulier là où fut construit le tohua de Kamuihei, parallèlement au versant. Ce choix est fréquent, pour les grandes structures notamment, afin de faciliter le travail d'aplanissement du terrain et d'apport des matériaux de construction. La paroi rocheuse qui le domine est bien visible depuis la cour et les gradins. On se sent vraiment, là, sous la « protection » de Te Moui.

De l'autre côté du torrent, à l'ouest, le me'ae de Tei'ipoka comprend trois paepae alignés, impressionnants, dont un supporte un banian majestueux ao'a (Ficus prolixa, var. marquesensis), l'un des plus grands des Marquises. Ce me'ae est séparé du tohua, proche, par le lit creusé du torrent. Ce qui n'empêche pas le tohua d'être, topographiquement même, placé sous les auspices du me'ae ; une cérémonie pouvait aisément se dérouler de part et d'autre du torrent, débuter par exemple sur le tohua pour se terminer sur le méae.

Ces trois paepae sacrés se distinguent, outre la présence du grand banian et leur position dominante, par l'existence de fosses reliquaires dans deux d'entre eux et, pour le troisième, par trois niveaux de pavage, chiffre au symbolisme important à rapprocher notamment des trois divinités des chefs : Teuutoka, Teuuhua et Tehitikaupeka. L'utilisation de dalles de grès de plage de couleur blanche, couleur réservée au monde religieux et funéraire, est également remarquable, d'autant plus qu'il n'en existe pas de cette teinte sur le littoral de Hatiheu et qu'il faut aller les chercher, en pirogue, sur la plus éloignée des plages de Anaho, à l'est, près de Ha'atuatua.

En contrebas du me'ae, toujours à l'ouest du tohua et dans son prolongement exact d'ailleurs, se trouve un très grand paepae, de $20 \mathrm{~m}$ sur $12 \mathrm{~m}$. Il n'est pas orienté comme de coutume vers le bas de la pente, mais vers le haut, car il s'oriente vers l'espace sacré (me'ae) mais à bonne distance ! Le terrain qui les sépare est libre d'aménagements lithiques notoires, mais compte plusieurs rochers émergeant du sol dont beaucoup sont ornés de

5. Les fosses à $m a$, souvent circulaires, peuvent être simplement creusées dans un sol ferme, c'est le cas le plus courant, ou parementées (pakeho). Dans ce cas, celles observées en particulier à Ua Pou, sont carrées. Les fosses familiales, selon les vallées et l'importance de l'unité d'habitation, ont de un à deux mètres de diamètre et à peu près autant de profondeur. Il existe de grandes fosses communautaires, qui font penser à de grands trous d'obus, dont les dimensions tournent autour de cinq à dix mètres de diamètre pour trois à quatre mètres de profondeur. La côte nord de Nuku Hiva en particulier, dont Hatiheu, compte un très grand nombre de fosses maçonnées dans les terrasses avant de paepae. Il pourrait s'agir de réserve, ou murissoir, selon certains, disons de petites fosses "alimentaires » destinées aux maisonnées. Il existe aussi, notamment sur l'un des paepae particulier de Kamuihei, des fosses circulaires, de plus de deux mètres de profondeur sur un peu plus d'un mètre de large, qui servirent de « fosse à prisonnier ». Ces exemples n'épuisent pas la typologie des fosses marquisiennes qui reste largement à faire. 


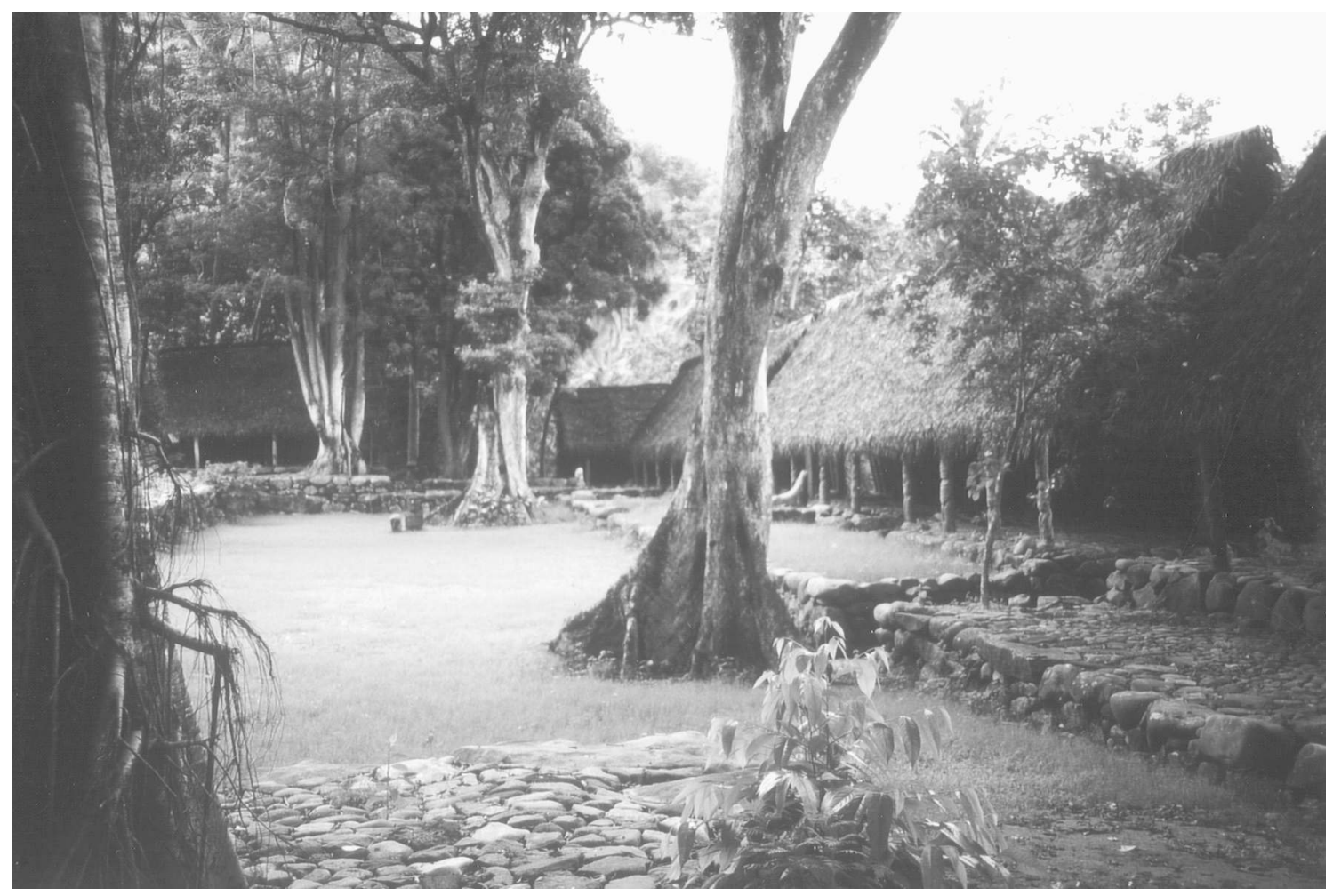

Photo 1. - Le tohua de Koueva, à Taiohae.

Vue de la cour après la restauration des structures lithiques, surmontées, à l'arrière, des ha'e construits en matériaux végétaux, qui abritaient les participants et les visiteurs. Long de $95 \mathrm{~m}$ et large de $30 \mathrm{~m}$, ce tohua est situé le plus en hauteur des cinq autres que comportait la vallée. Les racines, visibles sur la gauche, sont celles d'un grand banian placé à une extrémité de la cour, qui porte également deux grands badamiers, marquant les entrées principales de la place.

pétroglyphes. L'association entre ce très grand paepae (sans doute maison de chef ou maison des guerriers, lieu de réunion et de décisions) et un vaste espace communautaire tout à côté, avec un site religieux qui le domine, est intéressante mais pas unique. Elle souligne le caractère particulier de cette construction imposante, très soignée, et ornée d'un long alignement de ke'etu (tuf volcanique taillé en dalle) qui était réservé aux plus belles constructions. La place importante qu'elle occupe visuellement reflète sans aucun doute celle qu'elle tenait dans la société, à mi-chemin entre sacré et profane, entre un lieu de festivités et d'échanges et un lieu de décisions, à la fois point de contact avec les hommes et lien avec les divinités.

La restauration de ce paepae et la construction du $h a$ 'e qui le recouvrait permit, entre autres, de «mesurer» la masse de matériaux nécessaires. Pour la simple couverture du ha'e de $20 \mathrm{~m}$ de long, $6 \mathrm{~m}$ de haut et $5 \mathrm{~m}$ de large, il fallut 4000 palmes de cocotier tressées. Or, l'usage le plus courant autrefois dans l'archipel était plutôt de couvrir les bâtiments de petits paquets de feuilles d'arbre à pain... Là, il serait plutôt question de dizaines de milliers de feuilles à préparer, sans compter la masse basaltique qu'il fallut déplacer et agencer, la quantité de bois qu'il fallut préparer, pour la charpente, les centaines de mètres de fibres pour les ligatures... De tels efforts, les problèmes architecturaux qu'ils accompagnent, donnent idée de la qualité d'une main d'œuvre bien entraînée par d'autres travaux d'envergure, qui n'ont pas manqué dans cette vallée.

À l'arrière des trois paepae sacrés du me'ae, qui sont encore en liaison étroite avec le monde des hommes et des guerriers qu'ils dominent et auxquels ils font face, s'étage l'autre pan du méae: un autre monde, concrètement "à l'écart» et plus élevé encore. À remarquer à nouveau, qu'à mesure que le terrain s'élève, on passe de lieux voués, en contrebas, à l'alimentaire puis au quotidien en progressant vers des espaces constituant le cœur social de la communauté : le tohua et son lieu de réunion où se rassemblaient chefs et guerriers, pour aboutir enfin à l'espace sacré où était ravivé le lien avec les divinités par l'intermédiaire des personnes séjournant aux points dominants de ce territoire. 


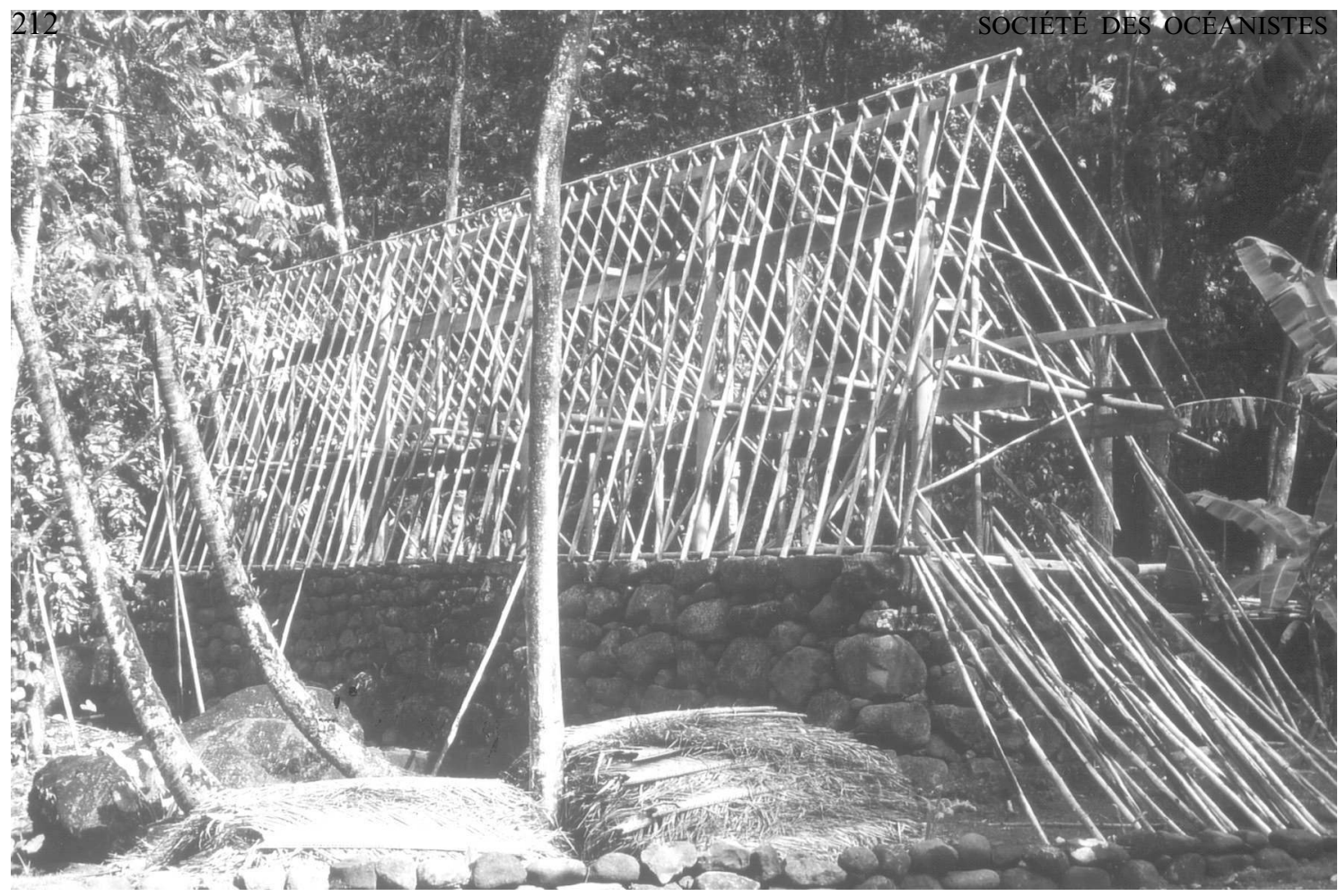

Рното 2. - Vue arrière du grand paepae de Kamuihei.

De $20 \mathrm{~m}$ de long sur $12 \mathrm{~m}$ de large, ce paepae était sans doute un des plus importants de l'ensemble et devait être réservé aux chefs et aux guerriers. Après sa restauration, la charpente du ha'e a été reconstruite avec des troncs de cocotiers (hauts de $6 \mathrm{~m}$ pour les plus grands, les pou), de badamiers et d'arbres à pain ; les chevrons sont constitués de perches d'hibiscus stériles (hau he'e) sur lesquels seront attachés, avec des écorces d'hibiscus (hau ki'i), les demi-palmes de cocotier tressées ( $\left.p o^{\prime} a\right)$ dont une partie repose au sol.

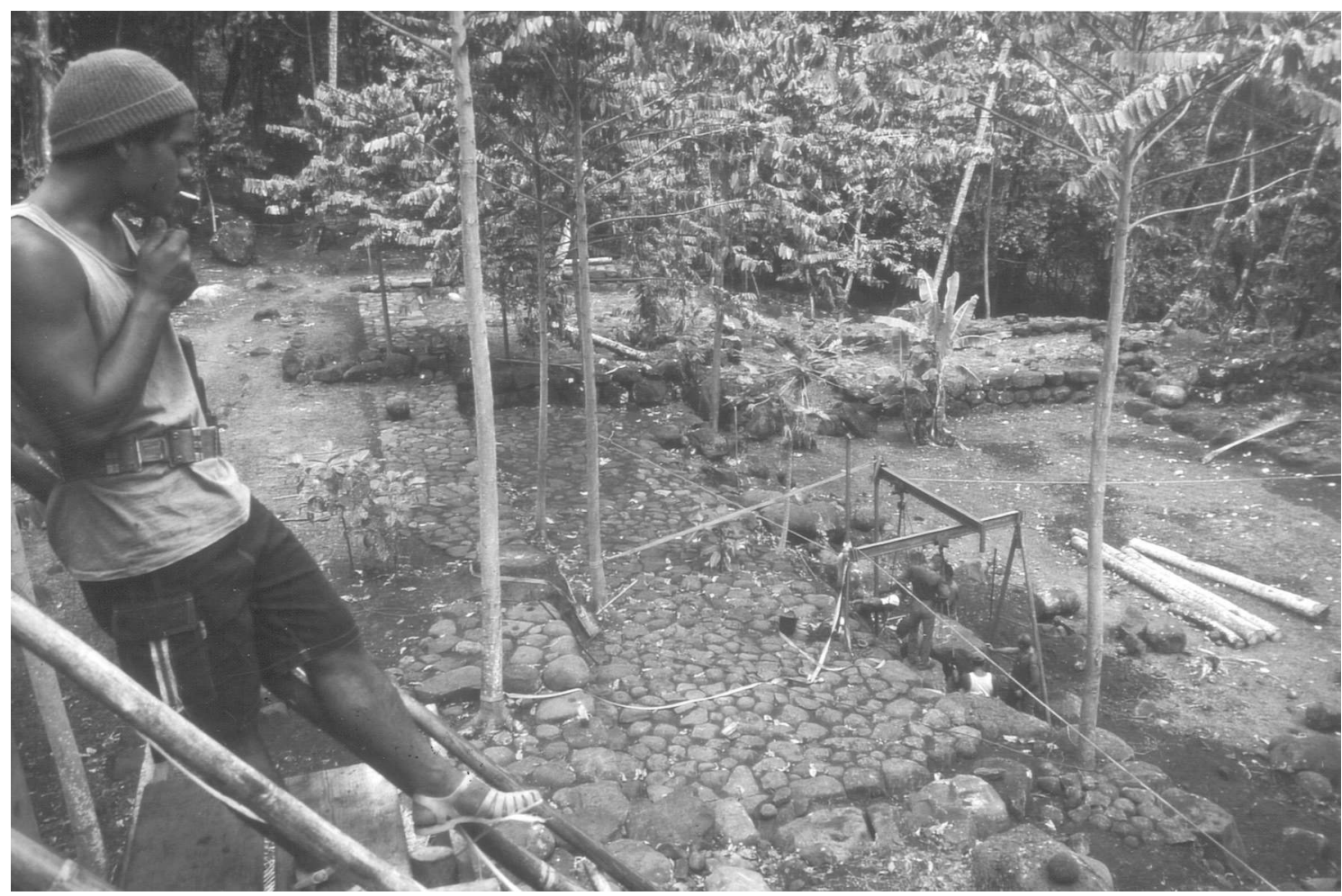

Рното 3. - Vue partielle du tohua de Kamuihei.

Une partie des paepae et des gradins a été restaurée. Il reste encore le mur soutenant le premier gradin le long de la cour où sont placés les portiques, utilisés pour la mise en place plus aisée des lourds blocs de basalte; des troncs de cocotiers ont été réservés pour les petits poteaux frontaux des futures habitations. Tonato apprécie la vue du sommet d'un des $h a^{\prime} e$ en cours de construction. 
Ici, à la base du promontoire de Te Moui, deux banians apportent encore leur ombre, considérée autrefois comme sacrée, à l'extrémité d'un aménagement qui se présente comme un petit tohua rectangulaire délimité, côté montagne, par des gradins et, côté mer, par des paepae diversement alignés, témoignant de l'évolution du lieu au cours des temps ${ }^{6}$.

$\mathrm{Au}$ nord-ouest se trouve un amas rocheux orné de pétroglyphes. Les figures d'un des rochers sont surtout anthropomorphes; l'autre rocher s'organise autour d'animaux marins: grands poissons et tortues, soit la représentation, rare aux Marquises, d'un animal prestigieux passant de la mer à la terre pour y confier sa progéniture qui, une fois éclose, n'aura de hâte que de regagner l'océan. Cet animal traduisait par excellence la notion de passage d'un monde à l'autre, entre la terre et la mer, entre le monde des vivants et celui des ancêtres, entre le monde des hommes et celui des divinités ; animal remarquable dont la capture était réservée aux chefs et dont le sacrifice équivalait à celui d'un être humain. Sa consommation était réservée aux dieux, aux prêtres et aux chefs. La localisation de ces motifs, dans la partie la plus élevée du site, juste à la base des flancs de Te Moui, ajoute à leur importance et au rôle de médiateur qu'ils jouèrent probablement entre les hommes et les puissances ancestrales divines.

À l'est, à très peu de distance, se trouve un grand 'ua ma, le plus important de l'ensemble et le plus élevé de Kamuihei, directement au pied du piton. Divers éléments font de ce lieu de réunion un espace particulier. Ses dimensions sont étonnamment " intimes », dans le contexte environnant, avec $58 \mathrm{~m}$ sur $26 \mathrm{~m}$ seulement; des mesures qui pourraient être plus habituelles sur d'autres îles, plus petites, comme Ua Huka par exemple. Ici, par contre, il ne pouvait s'agir d'y accueillir l'ensemble du clan. Par ailleurs, il compte un nombre remarquable de pierres à cupules - de dix centimètres de diamètre sur à peu près la moitié de profondeur —, souvent assemblées par paires, certaines pierres en regroupant de très nombreuses - de six à dix. Ceci dénote une activité liée à des gestes ou préparations sortant du quotidien, tels des remèdes, charmes ou onguents.

La présence de monticules de pierre d'assez petites dimensions, sur la pente amont, rappelle des plates-formes de traitement des corps. Enfin, les bancouliers 'ama (Aleurites triloba) abondent à cet endroit et leur noix fournissaient non seulement la lumière et le pigment servant à la pré- paration de l'encre du tatouage, mais elles étaient aussi connues pour leur propriété à fournir une huile desséchant la peau... effet et association symbolique recherchés dans la conservation des corps. Ces structures quadrangulaires effondrées, destinées probablement à des activités funéraires, se mêlent aux coulées de pierrailles qui endommagèrent l'endroit ; elles furent laissées telles quelles. Ce petit tohua fut en effet construit à la limite du raisonnable : le plus haut possible sur les terrains aménageables et il en pâtit. Les ruissellements et les colluvions comblèrent progressivement sa cour, des paepae virent leurs murs disparaître sous les dépôts de pente et des murets furent élevés pour retenir cet apport incessant. Ceci traduit-il un changement climatique ou un dérèglement saisonnier d'une ampleur supérieure à la moyenne ? En mai 1999, la seconde compagnie du Rimapp dégagea les sédiments sur parfois plus d'un mètre d'épaisseur pour retrouver les anciennes structures enfouies.

Ainsi, compte tenu de sa situation, des aménagements qui l'environnent et des usages plaçant toute grande activité sous le patronage des divinités, nous aurions tendance à voir ici un lieu voué à des réunions d'une portée plus restreinte, à caractère plus sacré, et fréquenté par des prêtres, ou personnes placées sous l'effet du tapu qui assuraient un lien entre une fonction, une situation sociale et des ancêtres divinisés.

\section{Conclusion}

Cette répartition des structures traduit une organisation spatiale réfléchie, harmonieuse, judicieuse et adaptée à des considérations religieuses aussi bien que de terrain. Tous les aménagements nécessaires à la vie d'une communauté y sont pratiquement réunis. Ce large éventail, la complémentarité des structures, leurs dispositions et les réaménagements montrent que la plupart furent sans doute en fonction à la même époque, dont la dernière période qui connut l'arrivée des Européens.

Par contre, cette mise en place prit des siècles avant d'aboutir à une telle densité et l'image qu'elle nous offre est l'aboutissement d'un lent processus qui s'élabora entre une société, avec ses concepts, son imaginaire et l'environnement.

Le Territoire dispose là d'un lieu, parmi d'autres certes, particulièrement remarquable à tout point de vue. Mais ces sites ne furent restaurés qu'en partie et doivent rester, si possible, le

6. La tradition y garde le souvenir d'une femme de 'A'akapa, capturée et torturée sur le plus ancien banian du lieu; des crânes y furent découverts en 1999. 
support d'autres efforts de conservation (notamment botaniques), de mise en valeur et de recherche à titre pédagogique, écologique, touristique et bien sûr archéologique, dans l'optique peutêtre d'une vision nouvelle du patrimoine, qui se met en place en plusieurs points du monde, et permet à des communautés de maintenir un mode de vie et un environnement en harmonie avec un patrimoine, pour sa sauvegarde et la nôtre. Ces sites n'ont pas été aménagés pour les touristes, mais ces derniers viennent parce que des communautés y ont accompli des réalisations remarquables. Aux touristes de s'adapter aux conditions du pays et au caractère de ses habitants, en ce qu'ils ont de bon sens, plutôt que l'inverse.

\section{RÉFÉRENCES}

Millerström S., 1990. Rock Art of the Marquesas Islands, French Polynesia : A Case Study of Hatiheu Valley, Nuku Hiva. M.A. Thesis, n.p., San Francisco State University.

SugGs R.C., 1961. «Archaeology of Nuku Hiva, Marquesas Islands, French Polynesia, Anthropological Papers of the American Museum of Natural History, $\mathrm{n}^{\circ} 49$ (1), New York, American Museum of Natural History. 